\section{Temperature-Dependent Kink Effect Model for Partially-Depleted SOI NMOS Devices}

\author{
S. C. Lin and J. B. Kuo
}

\begin{abstract}
This paper reports a closed-form analytical temperaturedependent kink effect model for the partially-depleted SOI NMOS devices. Based on the body-emitter voltage model, an analytical triggering $V_{\mathrm{DS}}$ formula for temperature-dependent kink effect has been obtained. According to the analytical model, at a higher operation temperature and with a lighter thin-film doping density, the onset of the kink effect occurs at a larger $V_{\mathrm{DS}}$.
\end{abstract}

\section{INTRODUCTION}

Partially-depleted SOI NMOS devices have been intensively studied [1]-[3]. Due to the floating body structure, kink effect is an important phenomenon for partially-depleted SOI NMOS devices. The kink effect can be explained as follows. In partially-depleted SOI NMOS devices, owing to the existence of the neutral region in the body, the source-body potential barrier is large. Therefore, the generated holes due to impact ionization are easily trapped in the body. Consequently, the potential barrier between the source and the body decreases. The hole current generated by impact ionization flows from body to source. Due to the increase in the body potential, the threshold voltage changes depending on the drain bias-the kink effect [4], [5]. Recently, CMOS circuits such as dynamic logic gates, frequency dividers, and operational amplifier realized by SOI CMOS devices operating at an elevated ambient temperature have been reported [6]. Analysis of the SOI MOS devices operating at an elevated ambient temperature has also been reported [7]-[9]. At an elevated ambient temperature, kink effect is still important. Analysis of the kink effect for the partially-depleted SOI NMOS device has been reported [9]. However, it's not closed-form. In order to gain insights, a closed-form analytical temperature-dependent kink effect model is derived in this paper. In the following sections, the closed-form analytical model is derived first, followed by the model verification and discussion.

\section{Model Derivation}

For a partially-depleted SOI NMOS operating at high temperature, biased in the saturation region there are unsmooth transitions in the drain current due to impact ionization. This is the so-called kink effect [4], [5]. At any operating temperature, at the kink, the body-emitter voltage of the parasitic bipolar device $\left(V_{\mathrm{BE}}\right)$, which is the difference in the quasi-Fermi potential across the body-emitter junction [10] $\left(V_{\mathrm{BE}}=\phi_{p}-\phi_{n}\right.$, where $\phi_{p}$ and $\phi_{n}$ are the hole and the electron quasiFermi potential at the body-emitter junction, respectively), has an abrupt change. The sudden increase in the body-emitter voltage at the kink in the saturation region is due to the accumulated holes caused by the injection of the hole current generated by impact ionization. At an elevated operating temperature, the kink effect is reduced. In

Manuscript received April 6, 1998. This work was supported under R.O.C. National Science Contracts NSC87-2215-E002-033 and NSC86-2622-E0002027. The review of this brief was arranged by Editor J. M. Vasi.

The authors are with the Department of Electrical Engineering, National Taiwan University, Taipei 106-17, Taiwan, R.O.C. (e-mail: jbkuo@cc.ee.ntu.edu.tw).

Publisher Item Identifier S 0018-9383(99)00273-7. this section, the temperature-dependent kink effect model is derived. In the following derivation, the temperature-dependent models for intrinsic concentration, electron mobility, generation/recombination lifetimes, and impact ionization are described subsequently, followed by the temperature-dependent current conduction mechanism.

\section{A. Temperature-Dependent Parameters}

For a partially-depleted SOI NMOS device, due to the neutral region in the thin film, it can be regarded as a combination of the MOS in top portion and the parasitic BJT in the bottom portion of the thin-film. When deriving the temperature-dependent model, both the top MOS and bottom parasitic bipolar effects should be included. There are four key temperature-dependent parameters used in the partially-depleted SOI NMOS device: intrinsic concentration, electron mobility, generation/recombination lifetimes, and impact ionization coefficients

1) Intrinsic Concentration: When the operating temperature rises, the bandgap of the silicon narrows. As a result, the intrinsic concentration increases. The temperature-dependent intrinsic concentration can be expressed as [11]

$$
\begin{aligned}
E_{g} & =1.17-\frac{4.73 \times 10^{-4} T^{2}}{T+636} \\
n_{i} & =4.9 \times 10^{15}\left(\frac{m_{n} m_{p}}{m_{o}^{2}}\right)^{0.75} T^{1.5} \exp \left(\frac{-E_{g}}{2 k T}\right)
\end{aligned}
$$

where $k$ is the Boltzmann's constant, $T$ is the operating temperature in Kelvins, $m_{n}$ and $m_{p}$ are the effective mass of the electron and the hole, respectively $\left(m_{n}=m_{o}\left(1.034+5.826 \times 10^{-4} T-2.826 \times\right.\right.$ $\left.\left.10^{-7} T^{2}\right), m_{p}=m_{o}\left(0.552+1.1 \times 10^{-3} T-7.769 \times 10^{-7} T^{2}\right)\right)[12]$. When the intrinsic concentration varies as the operating temperature rises, the related Fermi-potential and the depletion width in the device may change.

2) Electron Mobility: Electron mobility is dependent on the doping density and the operating temperature. Considering the surface reduction factor $\left(g_{s}\right)$ due to the surface scattering effect, the temperature-dependent electron mobility can be expressed as a function of the lattice mobility $\left(\mu_{l}\right)$ and the impurity mobility $\left(\mu_{i}\right)$ as [13]

$$
\begin{aligned}
\mu_{\mathrm{eff}} & =g_{s}\left(\frac{1}{\mu_{l}}+\frac{1}{\mu_{i}}\right)^{-1} \\
\mu_{l} & =\mu_{l 0}\left(\frac{T}{300}\right)^{-2.2} \\
\mu_{i} & =\frac{A T^{1.5}}{N_{A}}\left[\ln \left(1+\frac{B T^{2}}{N_{A}}\right)-\frac{B T^{2}}{N_{A}+B T^{2}}\right]^{-1}
\end{aligned}
$$

where $A=3.5 \times 10^{17} \mathrm{~cm}^{-1} \mathrm{~V}^{-1} \mathrm{~s}^{-1} \mathrm{k}^{\frac{-3}{2}}$ and $B=1.52 \times 10^{15}$ $\mathrm{cm}^{-3} \mathrm{k}^{-2}$ are dependent on the doping density. From simulation results, the surface reduction factor due to the surface scattering effect is $g_{s}=0.5$. At an elevated operating temperature, the lattice mobility $\left(\mu_{l}\right)$, which is the dominant factor, decreases.

3) Generation/Recombination Lifetimes: For a bipolar device, the generation/recombination lifetimes are important. In the bipolar device, the electron lifetime in the neutral region $\left(\tau_{n}\right)$, the generation lifetime $\left(\tau_{g}\right)$ and the recombination lifetime $\left(\tau_{r}\right)$ in the depletion region affect the leakage current of the device. The temperature- 
dependent electron lifetime, generation lifetime, and recombination lifetime can be expressed as [14]

$$
\begin{aligned}
\tau_{n} & =\tau_{n 0}\left(\frac{T}{300}\right)^{2.2} \\
\tau_{r} & =2 \tau_{n 0}\left(\frac{T}{300}\right)^{2.5} \\
\tau_{g} & =a_{\tau} T^{2.55} \cosh \left(\frac{b_{\tau}}{T}\right)
\end{aligned}
$$

where $\tau_{n 0}$ is the electron lifetime at $300 \mathrm{~K}$ and $a_{\tau}=3.5 \times 10^{-17}$ and $b_{\tau}=20$ are process-dependent parameters. When the operating temperature rises, the lifetimes increase. Consequently, the diffusion length $L_{e B}=\sqrt{\frac{k T}{q} \mu_{\mathrm{eff}} \tau_{n}}$ is affected.

4) Impact Ionization Coefficient: For a partially-depleted SOI NMOS device biased in the saturation region, impact ionization in the front channel and the back channel with a high electric field is important. Considering the temperature-dependent impact ionization effect, the multiplication factors of the front channel and the back channel are

$$
\begin{aligned}
M & =1+\frac{\alpha}{\beta}\left(V_{\mathrm{DS}}-V_{D \mathrm{SAT}}\right) \exp \left(\frac{-\beta l}{\left(V_{\mathrm{DS}}-V_{D \mathrm{SAT}}\right)}\right) \\
M_{B} & =1+\frac{\alpha_{b}}{\beta_{b}}\left(V_{\mathrm{DS}}+\phi_{\mathrm{bi}}\right) \exp \left(\frac{-\beta_{b} l_{b}}{V_{\mathrm{DS}}+\phi_{\mathrm{bi}}}\right)
\end{aligned}
$$

where $l=\sqrt{\frac{t_{\mathrm{ox} 1 x_{c}}}{\epsilon_{\mathrm{ox}} / \epsilon_{\mathrm{si}}}}, x_{c}=\sqrt{\frac{2 \epsilon_{\mathrm{si}}^{2} \phi_{f}}{q N_{A}}}, l_{b}=F_{c} \sqrt{\frac{2 \epsilon_{\mathrm{si}} V_{\mathrm{DS}}}{q N_{A}}}, \epsilon_{\mathrm{si}}$ is the permittivity of the silicon, $\epsilon_{\mathrm{ox}}$ is the permittivity of the oxide, $t_{\mathrm{ox} 1}$ is the thickness of the front gate oxide, $F_{c}$ is a fitting parameter [4], [5], and $\phi_{\mathrm{bi}}=\frac{k T}{q} \ln \frac{N_{A} N_{D}}{n_{i}^{2}}$ is the built-in voltage. $\alpha=1 \times 10^{6}$. $\beta=2.8 \times 10^{6}+1.8 \times 10^{3} \mathrm{~T}$. When the operating temperature is elevated from the room temperature, the travelling electron in the channel is subject to an increase in thermal scattering. As a result, the capability of producing electron-hole pairs owing to collision with lattice is reduced. Therefore, the multiplication factors $\left(M, M_{B}\right)$ are lowered when the operating temperature is increased. Note that in the above equations, $\alpha, \beta, \alpha_{b}, \beta_{b}$ are temperature-dependent empirical constants [15].

\section{B. Current Conduction Mechanism}

When considering the current conduction in a partially-depleted SOI NMOS device, both the top MOS and the bottom parasitic BJT in the thin-film are important. When a partially-depleted SOI NMOS device is biased with $V_{\mathrm{GS}}>V_{T}$ and $V_{\mathrm{DS}}>0$, the thin-film region above the buried oxide can be neutral-the parasitic npn bipolar device cannot be neglected. Due to the floating body effect, the parasitic bipolar device may be biased in the forward active region. For the parasitic npn bipolar device with the body-collector junction reverse biased, in the body-collector junction region, it has a reverse biased current $\left(I_{R}\right)$, which is composed of the generation current $\left(I_{\text {gen }}\right)$ in the space charge region and the electron diffusion current $\left(I_{\text {diff }}\right)$ in the undepleted body region outside the space charge region [16]:

$$
\begin{aligned}
I_{R} & =I_{\text {gen }}+I_{\text {diff }} \\
I_{\text {gen }} & =\frac{q n_{i} A W_{\mathrm{bc}}}{2 \tau_{g}} \\
I_{\text {diff }} & =q \sqrt{\frac{D_{n}}{\tau_{n}}} \frac{n_{i}^{2}}{N_{A}} A
\end{aligned}
$$

where

$W_{\mathrm{bc}}$ depletion width of the body-collector junction $\left(W_{\mathrm{bc}}=\right.$ $\sqrt{\left.\frac{2 \epsilon_{\mathrm{Si}}\left(\phi_{\mathrm{bi}}+V_{\mathrm{DS}}\right)}{q N_{A}}\right)}$;

$D_{n} \quad$ diffusion coefficient in the neutral region;

A cross section of the current flow in the parasitic bipolar device $\left(A=W\left(t_{\mathrm{si}}-\sqrt{\frac{2 \epsilon_{\mathrm{Si}}^{2} \phi_{f}}{q N_{A}}}\right)\right)$;

$W \quad$ width of the device;

$t_{\mathrm{si}} \quad$ thickness of the thin-film.

When the operating temperature is elevated, due to the influence from the intrinsic carrier concentration, the diffusion current $\left(I_{\text {diff }}\right)$ becomes important in $I_{R}$. Across the forward biased body-emitter junction in the parasitic bipolar device, the conducting current $\left(I_{F}\right)$ is made of the recombination current $\left(I_{\text {reco }}\right)$ in the space charge region and the electron diffusion current $\left(I_{\mathrm{ES}}\right)$ [16]:

$$
\begin{aligned}
I_{F} & =I_{\text {reco }} \exp \left(\frac{V_{\mathrm{BE}}}{2 k T / q}\right)+I_{\mathrm{ES}} \exp \left(\frac{V_{\mathrm{BE}}}{k T / q}\right) \\
I_{\text {reco }} & =\frac{q n_{i} A W_{\mathrm{be}}}{2 \tau_{r}} \\
I_{\mathrm{ES}} & =q \sqrt{\frac{D_{n}}{\tau_{n}}} \frac{n_{i}^{2}}{N_{A}} A
\end{aligned}
$$

where $W_{\mathrm{be}}$ is the depletion width of the body-emitter junction ( $W_{\mathrm{be}}=\sqrt{\frac{2 \epsilon_{\mathrm{si}} \phi_{\mathrm{bi}}}{q N_{A}}}$. When $V_{\mathrm{BE}}$ is small, the first portion of the above equation dominates $I_{F}$. When $V_{\mathrm{BE}}$ is large, the second portion becomes dominant. In addition, when the operating temperature is raised, the diffusion current becomes important.

Considering the MOS portion of the partially-depleted SOI NMOS device, the threshold voltage is subject to the floating body effect. The accumulated holes near the body-emitter junction in the thin-film due to the parasitic bipolar device caused by the floating body increase the body-emitter voltage $\left(V_{\mathrm{BE}}\right)$. As a result, the threshold voltage is lowered. Considering this effect, the threshold voltage model $\left(V_{T}\right)$ and the conducting current through the front channel $\left(I_{\mathrm{CH}}\right)$ under the front gate oxide have been reported in [5].

In this subsection, the current conduction mechanisms for the MOS portion and the parasitic bipolar portion of the partially-depleted SOI NMOS device have been explained. In the next subsection, the temperature-dependent kink effect model is derived for the device biased in the triode region $\left(V_{\mathrm{DS}} \leq V_{D \mathrm{SAT}}\right)$ and the saturation region $\left(V_{\mathrm{DS}}>V_{D \mathrm{SAT}}\right)$.

1) Triode Region $V_{\mathrm{DS}} \leq V_{D \mathrm{SAT}}$ : Fig. 1(a) shows the current conduction mechanism of the partially-depleted SOI NMOS device biased in the triode region. In the front channel region near the drain, there is no impact ionization. Therefore, no holes are injected into the floating body. As a result, the body-emitter voltage $\left(V_{\mathrm{BE}}\right)$ is small. Consequently, the parasitic bipolar device does not turn on. Under this situation, the floating body can be regarded as two diodes connected back to back. At the body-emitter junction, which is forward biased, the conducting current is $I_{F}$. At the body-collector junction, which is reverse biased, the conducting current is $I_{R}$. Considering the impact ionization effect at the body-collector junction, the conducting current is magnified by a factor of $M_{B}$-the conducting current becomes $M_{B} I_{R}$. Considering the current conduction of the parasitic bipolar device $\left(I_{F}=M_{B} I_{R}\right)$, from (5)-(7), the body-emitter voltage can be obtained:

$$
V_{\mathrm{BE}}=\frac{2 k T}{q} \ln \left[\frac{-I_{\mathrm{reco}}+\sqrt{I_{\text {reco }}^{2}+4 I_{\mathrm{ES}} M_{B}\left(I_{\text {gen }}+I_{\text {diff }}\right)}}{2 I_{\mathrm{ES}}}\right] .
$$




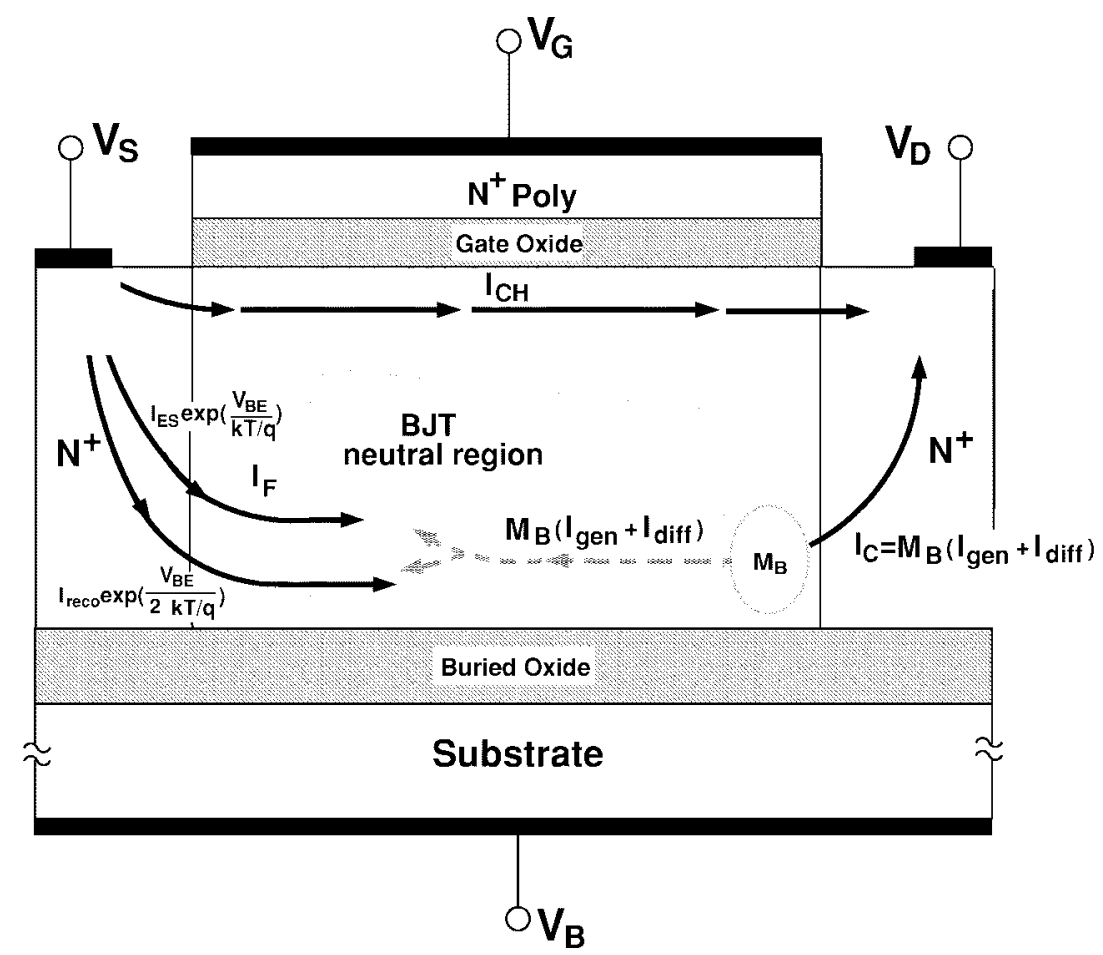

(a)

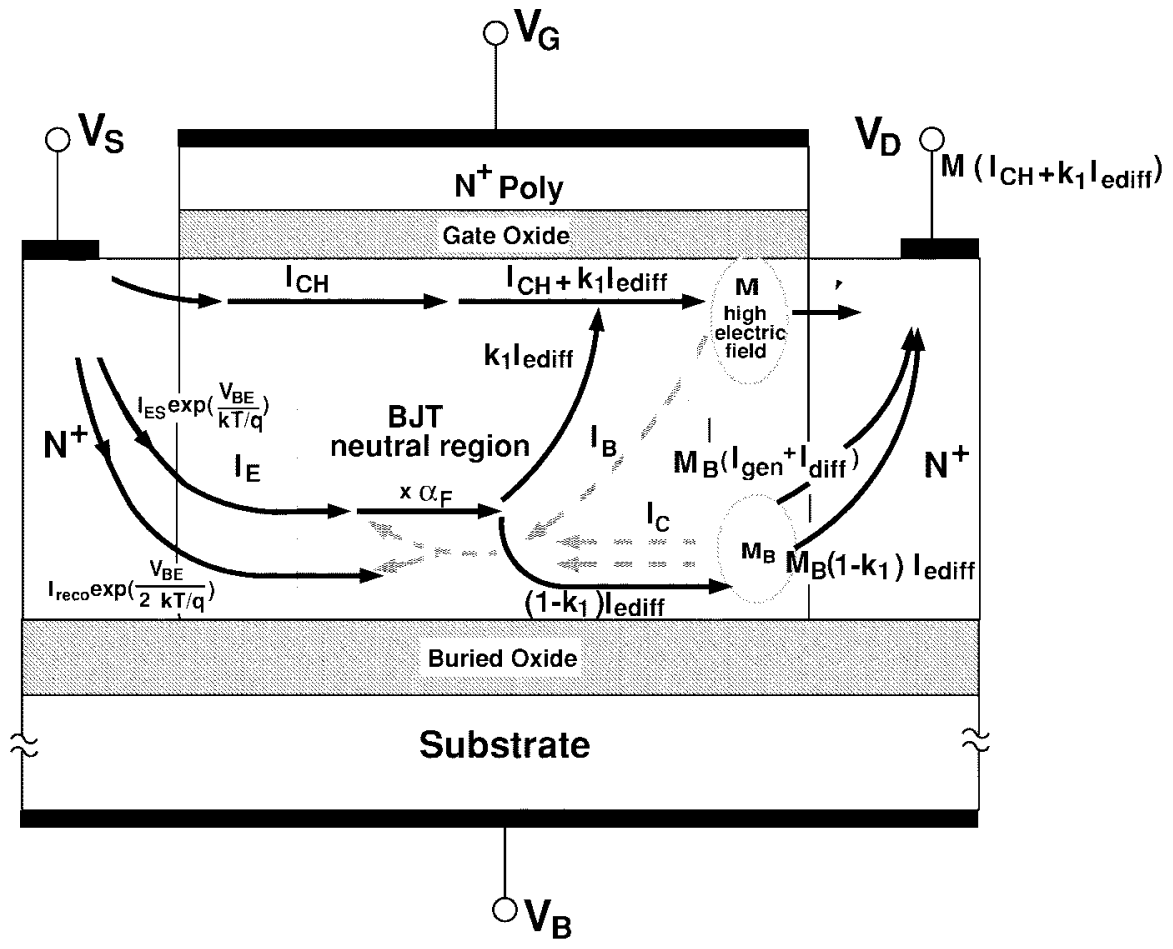

(b)

Fig. 1. The current conduction mechanism in the partially-depleted SOI NMOS device biased at (a) $V_{\mathrm{DS}} \leq V_{D} \mathrm{SAT}$ and (b) $V_{\mathrm{DS}}>V_{D \mathrm{SAT}}$.

In the above equation, the $\alpha_{b}$ and the $\beta_{b}$ used in $M_{B}$ are $\alpha_{b}=$ $7.5 \times 10^{5}$ and $\beta_{b}=3.41 \times 10^{6}+1.3 \times 10^{3} T[15]$.

2) Saturation Region $V_{\mathrm{DS}}>V_{D \mathrm{SAT}}$ : For the partially-depleted SOI NMOS device biased in the saturation region, the current conduction mechanism is shown in Fig. 1(b). In this region, due to $V_{\mathrm{DS}}>V_{D \mathrm{SAT}}$, the impact ionization in the front channel region near the drain is important. As a result, a large amount of holes due to the front channel impact ionization are injected into the floating body. Consequently, the accumulated holes near the body-emitter junction turn on the bottom parasitic bipolar device since the body-emitter voltage $V_{\mathrm{BE}}$ becomes large. From [5], in the saturation region, $V_{\mathrm{BE}}$ can be expressed as

$$
V_{\mathrm{BE}}=V_{\mathrm{BE} 1}+V_{\mathrm{BE} 2}-\left(V_{\mathrm{BE} 1}^{m}+V_{\mathrm{BE} 2}^{m}\right)^{1 / m}
$$


TABLE I

IMPORTANT PARAMETERS OF THE PARTIALLY-DePLeted SOI NMOS DEVICE UNDER STUdY

\begin{tabular}{lll} 
Symbol & Value & Unit \\
\hline$W$ & 50 & $\mu \mathrm{m}$ \\
$L$ & 25 & $\mu \mathrm{m}$ \\
$N_{A}$ & $1 \times 10^{17}$ & $\mathrm{~cm}^{-3}$ \\
$t_{o x 1}$ & 250 & $\AA$ \\
$t_{o x 2}$ & 4500 & $\AA$ \\
$t_{s i}$ & 2500 & $\AA$ \\
$\mu_{l 0}$ & 1500 & $\mathrm{~cm}^{2} / \mathrm{Vs}$ \\
$\tau_{n 0}$ & 0.9 & $\mathrm{~ns}$ \\
$F_{c}$ & 0.6 & \\
$m$ & 6 & \\
\hline
\end{tabular}

where $V_{\mathrm{BE} 1}$ is the $V_{\mathrm{BE}}$ when recombination current dominates the parasitic bipolar device; $V_{\mathrm{BE} 2}$ is the $V_{\mathrm{BE}}$ when diffusion current dominates the parasitic bipolar device. Based on the above analysis as shown in Fig. 1(a) and (b), the drain current of the partiallydepleted SOI NMOS device is composed of the front channel current in the front MOS portion and the collector current in the bottom parasitic bipolar device (see (10), shown at the bottom of the page). The above formulas are the closed-form analytical temperature-dependent kink effect model for the partially-depleted SOI NMOS device.

\section{Model Evaluation}

In order to verify the effectiveness of the analytical temperaturedependent kink effect model for the partially-depleted SOI NMOS device, the model results have been compared with the experimental data [9] and two-dimensional (2-D) simulation results. As shown in the table, the test device under study has a channel length of 25 $\mu \mathrm{m}$ and a channel width of $50 \mu \mathrm{m}$. The gate oxide is $250 \AA$. A thin-film of $2500 \AA$ is atop a buried oxide of $4500 \AA$. The doping density of the thin-film is $10^{17} \mathrm{~cm}^{-3}$. Fig. 2 shows (a) $V_{\mathrm{BE}}$ and (b) $I_{D}$ versus $V_{\mathrm{DS}}$ of the partially-depleted SOI NMOS device biased at $V_{\mathrm{GS}}=3.5 \mathrm{~V}$, based on the analytical model, the experimental data [9] [Fig. 2(b)] and the 2-D simulation results. As shown in Fig. 2(a), at all three operating temperatures, at the kink the bodyemitter voltage of the parasitic bipolar device has an abrupt change. As described in the previous section, the sudden increase in the body-emitter voltage at the kink in the saturation region is due to the accumulated holes caused by the injection of the hole current generated by impact ionization. As shown in the figures, at an elevated operating temperature, the kink effect is reduced. As verified by the experimental data [Fig. 2(b)] and the 2-D simulation results, the analytical model provides a good prediction of the temperaturedependent kink effect behavior of the partially-depleted SOI NMOS device. As shown in the figure, at a small $V_{\mathrm{DS}}$, the model result on $V_{\mathrm{BE}}$ is deviated from the simulation results due to the omission of the $V_{\mathrm{BE}}$ term from $W_{\mathrm{bc}}=\sqrt{\frac{2 \epsilon_{\mathrm{Si}}\left(\phi_{\mathrm{bi}}+V_{\mathrm{DS}}-V_{\mathrm{BE}}\right)}{q N_{A}}}$ as indicated in the sentence below.

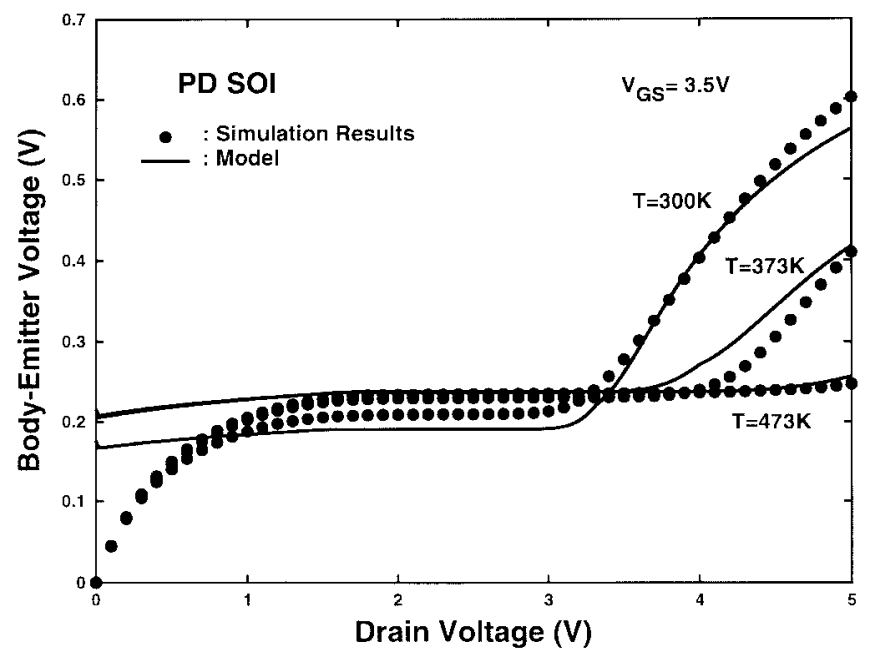

(a)

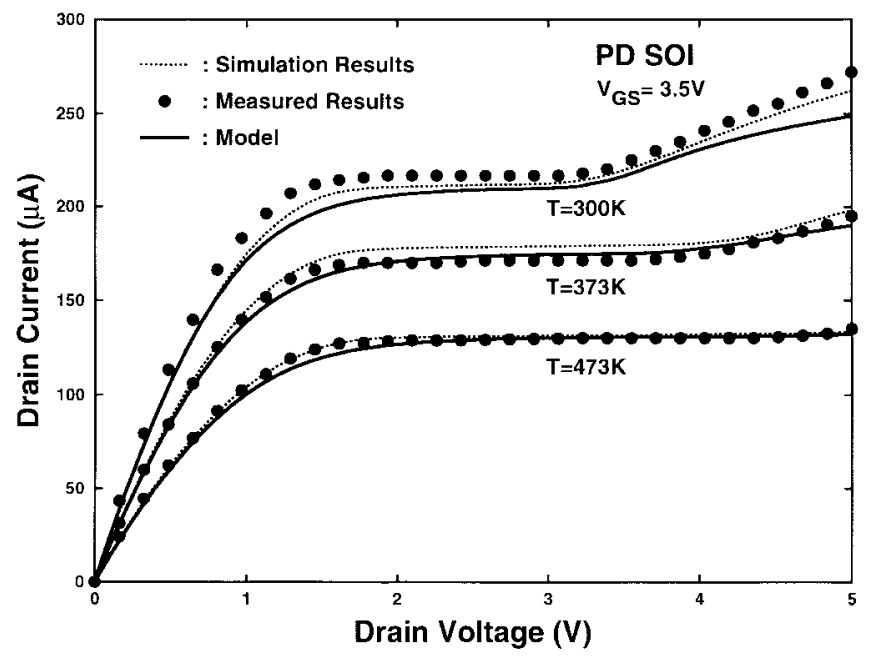

(b)

Fig. 2. (a) $V_{\mathrm{BE}}$ (b) $I_{D}$ versus $V_{\mathrm{DS}}$ of the partially-depleted SOI NMOS device based on the analytical model, the experimental data [9] (b) and the two-dimensional (2-D) simulation results.

\section{DISCUSSION}

The triggering $V_{\mathrm{DS}}$ at the onset of the kink effect is dependent on the operating temperature. Define $V_{\text {kink }}$ as the triggering $V_{\mathrm{DS}}$ when the kink effect is about to occur. At the onset of the kink effect, the body-emitter voltage is

$$
V_{\mathrm{BE}}=V_{\mathrm{BES}}+10 \mathrm{mV}
$$

where $V_{\mathrm{BES}}$ is $V_{\mathrm{BE}}$ at $V_{\mathrm{DS}}=V_{D \mathrm{SAT}}$. From [5], $V_{\text {kink }}$ is found (see (12), shown at the top of the next page). Based on (12), Fig. 3 shows the triggering $V_{\mathrm{DS}}\left(V_{\text {kink }}\right)$ at the onset of the kink effect versus the operating temperature of the partially-depleted SOI NMOS device for various thin-film doping densities, based on the analytical model. At an elevated operating temperature, $V_{\text {kink }}$ is increased-the kink effect is suppressed. With a higher doping density in the thin-film, $V_{\text {kink }}$ is lowered-the kink effect is more easily to occur since at a

$$
I_{D}= \begin{cases}I_{\mathrm{CH}}+M_{B}\left(I_{\text {gen }}+I_{\text {diff }}\right), & \text { for } V_{\mathrm{DS}} \leq V_{D \mathrm{SAT}} \\ \left(I_{\mathrm{CH}}+k_{1} \alpha_{F} I_{\mathrm{ES}} \exp \left(\frac{V_{\mathrm{BF}}}{k T / q}\right)\right) M+M_{B}\left(I_{\mathrm{gen}}+I_{\mathrm{diff}}+\left(1-k_{1}\right) \alpha_{F} I_{\mathrm{ES}} \exp \left(\frac{V_{\mathrm{BF}}}{k T / q}\right)\right), & \text { for } V_{\mathrm{DS}}>V_{D \mathrm{SAT}}\end{cases}
$$




$$
\begin{aligned}
V_{\mathrm{kink}}= & V_{D \mathrm{SAT}}+\frac{-D_{2}+\sqrt{D_{2}^{2}-4 D_{1} D_{3}}}{2 D_{1}} \\
D_{1}= & \ln \left(\frac{I_{\mathrm{reco}}}{I_{\mathrm{CH} 1}} \frac{\beta}{\alpha} \exp \left(\frac{V_{\mathrm{BES}}}{2 k T / q}\right)\left(e^{\frac{0.01}{2 k T / q}}-1\right)\right)-2 \\
D_{2}= & \ln \left(\frac{I_{\mathrm{reco}}}{I_{\mathrm{CH} 1}} \frac{\beta}{\alpha} \exp \left(\frac{V_{\mathrm{BES}}}{2 k T / q}\right)\left(e^{\frac{0.01}{2 k T / q}}-1\right)\right)+2+\beta l \\
D_{3}= & \beta l \\
I_{\mathrm{CH} 1}= & \frac{W}{L} \mu_{\mathrm{eff}} C_{\mathrm{ox}} V_{D \mathrm{SAT}} \\
& \times \frac{\left(V_{G}-V_{f b}-2 \phi_{f}-\gamma \sqrt{2 \phi_{f}-V_{\mathrm{BES}}-0.01}-\frac{1}{2}\left(1+\frac{\gamma}{2 \sqrt{\phi_{a}+2 \phi_{f}-V_{\mathrm{BES}}-0.01}}\right) V_{D \mathrm{SAT}}\right)}{1+\theta\left(V_{G}-V_{f b}-2 \phi_{f}-\gamma \sqrt{\left.2 \phi_{f}-V_{\mathrm{BES}}-0.01\right)-\theta_{B}\left(V_{\mathrm{BES}}+0.01\right)}\right.} .
\end{aligned}
$$

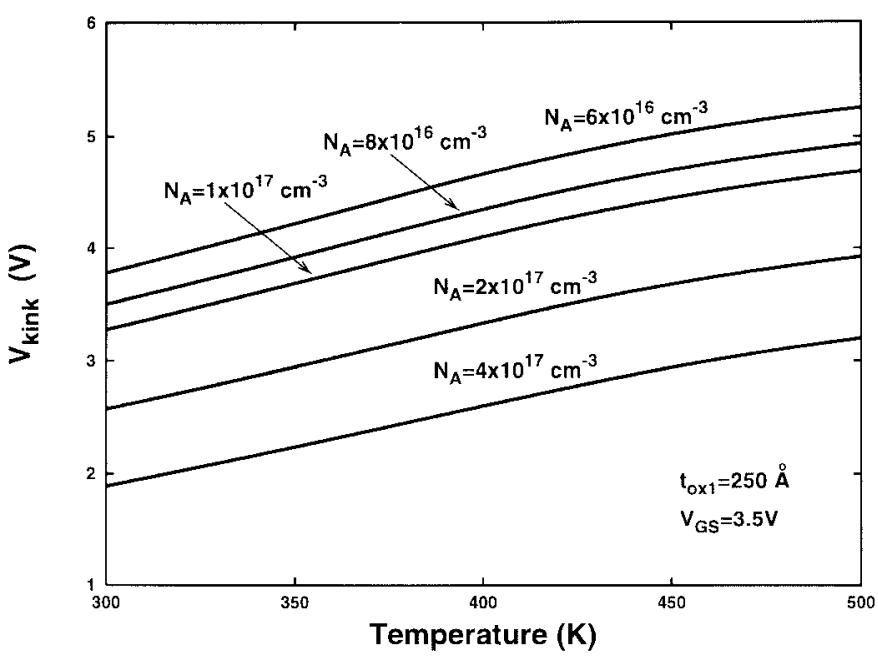

Fig. 3. The triggering $V_{\mathrm{DS}}\left(V_{\mathrm{kink}}\right)$ at the onset ofthe kink effect versus the operating temperature of the partially-depleted SOI NMOS device based on the analytical model for various thin-film doping densities.

higher doping density in the thin-film, the parasitic bipolar device is weaker. As a result, the impact ionization generated holes are less likely to be recombined in the base of the parasitic bipolar device. Instead, they are more likely to be accumulated at the body-emitter junction. As a result, the kink effect is more easily to occur.

\section{CONCLUSION}

In this paper, a closed-form analytical temperature-dependent kink effect model for the partially-depleted SOI NMOS devices has been described. Based on the body-emitter voltage model, an analytical triggering $V_{\mathrm{DS}}$ formula for temperature-dependent kink effect has been obtained. According to the analytical model, at a higher operation temperature and with a lighter thin-film doping density, the onset of the kink effect occurs at a larger $V_{\mathrm{DS}}$.

\section{REFERENCES}

[1] M. Matloubian, C.-E. D. Chen, B.-Y Mao, R. Sundaresan, and G. P Pollack, "Modeling of the subthreshold characteristics of SOI MOSFET's with floating body," IEEE Trans. Electron Devices, vol. 37, pp. 1985-1993, Sept. 1990.

[2] D. Suh and J. G. Fossum, "A physical charge-based model for nonfully depleted SOI MOSFET's and its use in assessing floating-body effects in SOI CMOS circuits," IEEE Trans. Electron Devices, vol. 42, pp. 728-737, Apr. 1995.
[3] K. Kato, T. Wada, and K. Taniguchi, "Analysis of kink characteristics in silicon-on-insulator MOSFET's using two-carrier modeling," IEEE Trans. Electron Devices, vol. ED-32, pp. 458-462, Feb. 1985.

[4] H.-K. Yu, J.-S. Lyu, S.-W. Kang, and C.-K. Kim, "A physical model of floating body thin film silicon-on-insulator nMOSFET with parasitic bipolar transistor," IEEE Trans. Electron Devices, vol. 41, pp. 726-732, May 1994.

[5] S. S. Chen and J. B. Kuo, "An analytical CAD kink effect model of partially-depleted SOI NMOS devices operating in strong inversion," Solid-State Electron., vol. 41, no. 3, pp. 447-458, Mar. 1997.

[6] P. Francis, A. Terao, B. Gentinne, D. Flandre, and J.-P. Colinge, "SOI technology for high-temperature applications," in IEDM Tech. Dig., 1992, pp. 353-356.

[7] G. Groeseneken, J.-P. Colinge, H. E. Maes, J. C. Alderman, and S. Holt, "Temperature dependence of threshold voltage in thin-film SOI MOSFET's," IEEE Electron Device Lett., vol. 11, pp. 329-331, Aug. 1990.

[8] D. Flandre, A. Terao, P. Francis, B. Gentinne, and J.-P. Colinge, "Demonstration of the potential of accumulation-mode MOS transistors on SOI substrates for high-temperature operation $\left(150-300{ }^{\circ} \mathrm{C}\right.$," IEEE Trans. Electron Device Lett., vol. 14, pp. 10-12, Jan. 1993.

[9] D.-S Jeon and D. E. Burk, "A temperature-dependent SOI MOSFET model for high-temperature application $\left(27^{\circ} \mathrm{C}-300{ }^{\circ} \mathrm{C}\right)$," IEEE Trans. Electron Devices, vol. 38, pp. 2101-2111, Sept. 1991.

[10] J. Y. Choi and J. G. Fossum, "Analysis and control of floating-body bipolar effects in fully-depleted submicrometer SOI MOSFET's," IEEE Trans. Electron Devices, vol. 38, pp. 1384-1391, June 1991.

[11] S. M. Sze, Physics of Semiconductor Devices. New York: Wiley.

[12] H. D. Barber, "Effective mass and intrinsic carrier concentration in silicon," Solid-State Electron., vol. 10, no. 6, pp. 1039-1051, 1967.

[13] J. M. Dorkel and Ph. Leturcq, "Carrier mobilities in silicon semiempirically related to temperature, doping and injection level," SolidState Electron., vol. 24, no. 9, pp. 821-825, 1981.

[14] D. K. Schroder, "The concept of generation and recombination lifetimes in semiconductors," IEEE Trans. Electron Devices, vol. ED-29, pp. 1336-1338, Aug. 1982.

[15] W. N. Grant, "Electron and hole ionization rates in epitaxial silicon at high electric fields," Solid-State Electron., vol. 16, pp. 1189-1203, 1973.

[16] A. Bar-Lev, Semiconductors and Electronic Devices. New York: Prentice-Hall, 1993. 